\title{
Cephalic Microstructure and its role in Predation Biology of Myrmicaria brunnea on Antheraea mylitta
}

\author{
Ganesh B. Gathalkar ${ }^{1 *}$, Deepak D. Barsagade ${ }^{2}$ \\ ${ }^{1}$ Laboratory of Entomology, Division of Organic Chemistry, CSIR-National Chemical Laboratory, Dr. Homi Bhabha Road, Pune- 411008, Maharashtra, \\ India. ${ }^{2}$ Department of Zoology, MJF Educational Campus, RTM Nagpur University, Nagpur -440033, Maharashtra, India.
}

\begin{tabular}{|c|c|}
\hline ARTICLE INFO & ABSTRACT \\
\hline $\begin{array}{l}\text { Article history: } \\
\text { Received on: } 06 / 07 / 2017 \\
\text { Accepted on: } 12 / 09 / 2017 \\
\text { Available online: } 17 / 01 / 2018\end{array}$ & $\begin{array}{l}\text { Antheraea mylitta (Drury) (Lepidoptera: Saturniidae) is commercially the most important species for the } \\
\text { world-class 'Wild Silk,' popularly known as 'Tasar' or 'Kosa' silk. However, the ant Myrmicaria brunnea } \\
\text { (Saunders) (Hymenoptera: Formicidae) is a serious predator of A. mylitta, and their predations include the } \\
\text { continuous pricking and biting leads to complete destruction of the silkworm larvae, and consequent crop }\end{array}$ \\
\hline $\begin{array}{l}\text { Key words: } \\
\text { Aggressive predator, invasions, } \\
\text { crop damage, feeding behavior, } \\
\text { tasar-culture. }\end{array}$ & $\begin{array}{l}\text { loss. Assuming the sensilla present on the antenna and mouthparts play an important role in predatory } \\
\text { success, we studied the distribution and diversity of sensilla by scanning electron microscopy (SEM). The } \\
\text { host-predator interactions based on this sensory physiology, will enable us to develop an effective control } \\
\text { strategy in sericultural practices, to trap this predator. }\end{array}$ \\
\hline
\end{tabular}

\section{INTRODUCTION}

Antheraea mylitta Drury (Lepidoptera: Saturniidae) produces a unique wild variety of "Tasar Silk," or "Kosa silk" [1, 2] and provides a livelihood security to many tribal families [3, 4]. During rearing, the wild tasar silkworm is exposed to the complexes of various parasites-predators, resulting into the loss of tasar silk production [5, 6]. However, among the predators, the ant Myrmicaria brunnea Saunders (Hymenoptera: Formicidae) is a tree dweller and forages among the tasar host plantation and has been identified as a larval predator of tasar silkworm A. mylitta [7]. The ant species are omnivorous, entomophagous, as well as sap-sucking and a ubiquitous component of tropical forests, which constitute a diversity of interaction with various flora and fauna [8]. The workers of M. brunnea form conspicuous columns and forage on the ground as well as the trees $[9,10]$ including the tasar host plants. During foraging, these ants (workers) attack the

\section{* Corresponding Author}

Laboratory of Entomology, Division of Organic Chemistry, CSIRNational Chemical Laboratory, Dr. Homi Bhabha Road, Pune-411008, Maharashtra,India.Email: ggathalkar@gmail.com;

Phone: +919764644826 larvae of A. mylitta in groups which lead to the complete larval destruction, resulting in larval mortality which ultimately reduces tasar silk production. The predation biology and predatory potential of $M$. brunnea on A. mylitta were investigated earlier [11].

Morphologically, the ant $M$. brunnea is relatively moderate in size, with a particular seven segmented geniculate type of antennae, shining mandibles and yellow body hairs [12-16]. However, in the present study, we examined the external morphology of $M$. brunnea with the help of scanning electron microscope (SEM). The SEM studies revealed the presence several machano- as well as chemoreceptors on the surface of antenna, mouthparts, and the sting apparatus which apparently play a significant role in host-location and feeding behavior of $M$. brunnea.

\section{MATERIAL AND METHODS}

\subsection{Insect source}

A survey was undertaken in the tasar rearing sites of Vidarbha, Maharashtra, where we observed the predatory attack of Myrmicaria brunnea on the larvae of A. mylitta (Fig. 1). During the predatory events, the insect predators were collected by hand picking method with the help of forceps and preserved in $70 \%$ alcohol for the further studies (i.e. SEM). 

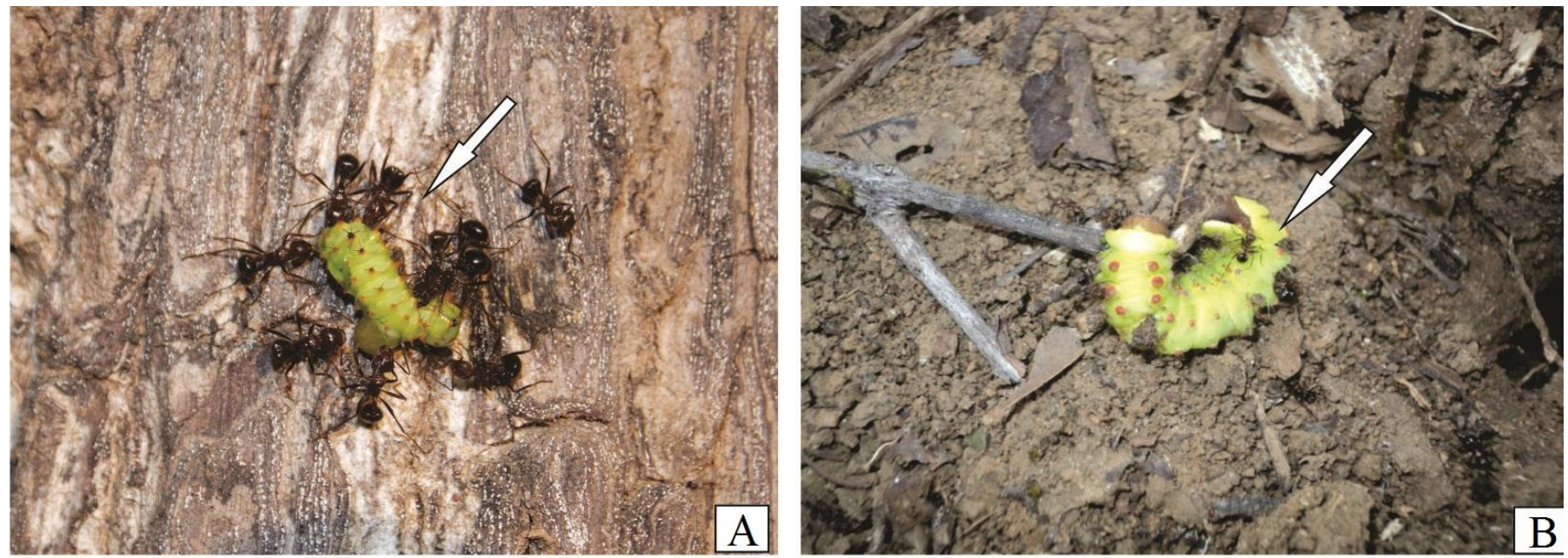

Fig. 1: The predatory ant, Myrmicaria brunnea (A-B) showing predatory attack on silkworm larva Antheraea mylitta (D).

\subsection{Morphological Preparation for Scanning Electron Microscopic (SEM) study}

The $70 \%$ alcohol preserved samples of ant $M$. brunnea were dissected under the stereoscopic binocular microscope (Carl Zeiss Stemi DV4), and various appendages such as the head, antennae, mouthparts, and the last abdominal segment were separated. The dissected parts were washed thoroughly and fixed in $10 \%$ formalin for $12 \mathrm{hrs}$. They were subsequently dehydrated in a series of alcohol grades, cleared in acetone $\left[(\mathrm{CH} 3)_{2} \mathrm{CO}\right]$, dried at room temperature, mounted on carbon-coated metallic stubs at different angles, and gold coated in a Poloron Gold Coating Automatic Unit. Specimens were observed with a JEOL JSM6380A Scanning Electron Microscope (SEM) at a desirable magnification at the Instrumentation Centre of Visvesvaraya National Institute of Technology (VNIT) Nagpur, India.

\section{RESULTS}

\subsection{Scanning electron microscopic (SEM) study of M. brunnea}

SEM study of the antenna, mouthparts and sting apparatus of worker ant M. brunnea revealed the presence of various sensillae.

\subsection{Antenna}

The antenna of $M$. brunnea is geniculate with seven segments. They appear elbow-shaped, consisting of a scape, a pedicel and five flagellomeres (Figs. $2 \mathrm{~A}-\mathrm{F}$ ).

\subsection{Types of sensilla \\ 3.3.1 Scapal sensilla}

The surfaces of the scape were covered with polygonal cuticular plates forming the cuticular micro-sculpture. Three types of sensilla basiconica, SB-I, SB-II and SB-III were observed on the posterodorsal region of the scape (Figs. 2A-D). The SB-I are smooth on the dorsal as well as the ventral surface, long and straight with a broad base and a pointed tip. The SB-II are arranged in four rows and are smaller than the SB-I. The shaft of the SB-II sensilla are smooth, pointed and projecting from a bulbous basal cuticular structure. The SB-III are short and are located ventral to the SB-II. The shaft of these sensilla are short, pointed and project from a broad, bulbous base.

The entire surface of the elongated and filamentous shaft of the scape, bears the micro-sculpture of polygonal cuticular plates. Along with these plates, two types of trichoid sensilla, ST-I, and ST-II were observed. The ST-I are long and originated from the large cuticular plates on the dorsoventral surface of the pedicel. The sensilla are pointed terminally and broad at the base. In contrast, ST- II are short, small in size and distributed throughout the entire surface.

\subsubsection{Pedicellar sensilla}

Trichoid sensillae are present throughout the surface of the pedicel in worker ants (Figs. 2 C-D).

\subsubsection{Flagellar sensilla}

Sensilla trichoidea curvata (STC) and sensilla trichoidea (ST) are present densely on the flagellar segments on dorsal as well as ventral surfaces. Besides, these two types of sensilla, the last two flagellar segments show two types of sensilla basiconica also. The shafts of STC are long, tapered and slightly curved apically, whereas those of the ST are long, narrow towards the apex and tapering terminally. The SB are short and were observed on the dorsoventral surface of the last flagellar segments of worker ants. The SC was concentrated on terminal flagellar segments at mid-dorsal and ventral surfaces in the male ants. Their shafts were long, flattened and tapered towards the terminal tip (Figs. 2 E-F). 

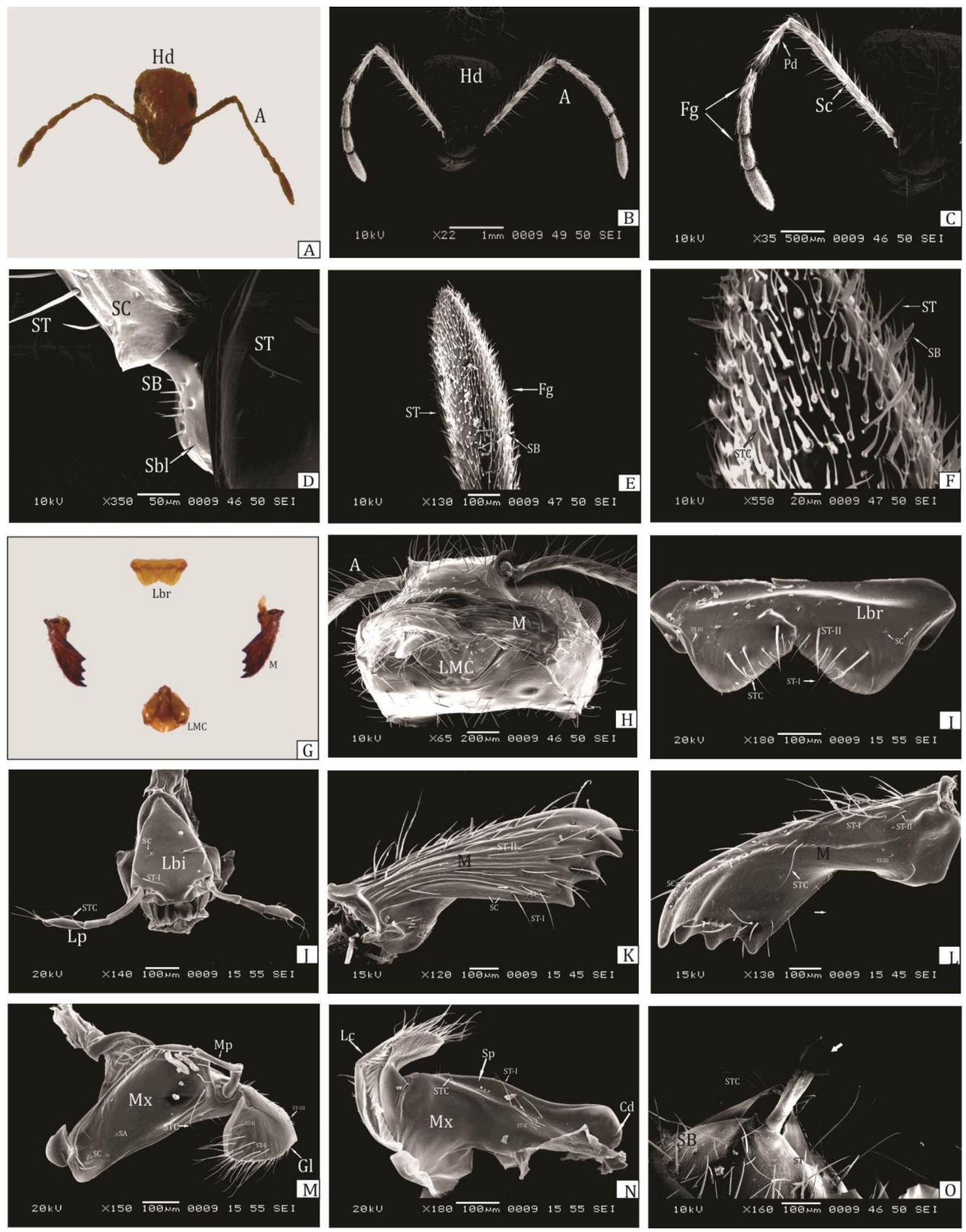

Fig. 2: Structure of M. brunnea showing A: Light microscopic view of the head with the antenna. Scanning electron microscopic structure showing, B: Head and antenna; C: Close view antenna with sensillae; D: Antennal socket with ball and scape with sensilla; E: Flagellum and F: Sensillae present on the flagellum. G-N: Mouthparts and scanning electron microscopic (SEM) structure of mouthparts showing, H: Front view of mouth with arrangements of mouthparts; I: Labrum; J: Labium; K: Dorsal view of mandible; L: Ventral view of mandible; M: Dorsal view of maxilla; N: Ventral view of mandible and O: Sting apparatus. Abr.: Hd-Head, A-Antenna, Sc- Scape, Sbl- Scape ball, Fg- Flagellum, Lbr- Labrum, Lbi- Labium, M-Mandible, Mx- Maxilla, LMC- Labio-maxillary complex, Mp- Maxillary palp, Lp- Labial palp, Cd-Cardo, SpStipes, Lc-Lacinia, Gl-Galea, ST- Sensilla trichoidea, STC- Sensilla trichoidea curvata, SB-Sensilla basiconica, SA- Sensilla ampulacea and SC- Sensilla coeloconica. 


\subsection{Mouthparts}

The mouth parts are adapted for biting and chewing of the food. The mouthparts consisted of the labrum, mandible, maxilla, and labium (Figs. 2 G-N).

\subsubsection{Labrum}

The labrum was movable and situated beneath the middle range of the clypeus and folds up under the closed mandibles forming the upper lip. Structurally, it is bilobed, flattened and each lobe is somewhat triangular in shape. It is yellowish brown in color with numbers of ancillary hairs on it (Fig.1 H-I).

\subsubsection{Sensilla of labrum}

The labrum revealed the presence of three types of sensilla on the labrum: sensilla trichoidea, sensilla trichoidea curvata, and sensilla coeloconica. Morphology categorizes the trichoid sensilla as ST-I, ST-II, and ST-III (Figs. 2 H-I).

\subsubsection{Labium}

The labium is the lower lip of the subterranean insect and frames the combination of a couple of appendages. The principle body of the labium is partitioned into three sections as the central 'mentum", "submentum" and the "prementum" distally. The prementum underpins two sets of flaps known as the "glossae" and to the outside of them the "paraglossae" and a pair of three sectioned labial palps which are tangible in capacity (Figs. $2 \mathrm{H}-\mathrm{I}$ ).

\subsubsection{Sensilla of labium}

Surface ultrastructure of labium uncovered the vicinity of sensory structures, including sensilla trichoidea, sensilla trichoidea curvata, and sensilla coeloconica. Additionally, on the labial palp sensilla trichoidea and sensilla trichoidea curvata were observed (Figs. $2 \mathrm{H}-\mathrm{I}$ ).

\subsubsection{Mandible}

The mandibles are unsegmented, sclerotized, large with a scoop like cuticular structure. The mandibles bear solid basal and almost equal sized three molars and one distal incisor teeth. The longitudinal edge or outskirt of each mandibular cutting edge are furnished with teeth near a front augmentation of the head's midline. The base of this edge is near the front edge of the clypeus (Figs. 2 G-H and K-L).

\subsubsection{Sensilla of the mandible}

On the dorsal and ventral surfaces of the mandibles, trichoid sensilla, basiconic sensilla, and sensilla coeloconica were observed. The trichoid sensilla are differentiated into ST-I, ST-II, and ST-III types. The sensilla ST-I are present on the marginal area of the dorsal region of mandibles.

The sensilla trichoidea ST-II are long, slightly curved, pointed while the ST-III are short and scattered throughout the dorsal surface. Sensilla coeloconica (SC) are observed on the upper peripheral territory of the mandible (Figs. 2 K-L).

\subsubsection{Maxilla}

These paired structures are homologous and composed with proximal cardo, middle stipes, lateral palpifer, inner parastipes of subgalea and distal sclerite consisting two lobes, inner lacinia, and the outer galea. Palfiper bears the maxillary palp, comprised of three segments and possesses hairs and bristles. The labium and the maxilla forms a labiomaxillary complex, and their palpus is easy to observe on the ventral side. On the ventral side of the head, the maxillae are situated within the oral cavity, on both sides of central labium. The basal segment of the maxilla attaches to the head capsule by the structure, cardo and stipes. The stipes bears a maxillary palp, an inner surface of the stipes bears a lobe towards its distal end, the lacinia. The free margin of lacinia is irregular and minutely dentiform. The distal tip of the stipes contains a terminal lobe known as galea, which often folds over the lacinia. Maxillary palp is three segmented and lies ventral to the maxilla (Figs. $2 \mathrm{M}-\mathrm{N}$ ).

\subsubsection{Sensilla of the maxilla}

The surface of the maxilla are endowed with sensilla trichoidea type-I, II, III, sensilla trichoidea curvata (STC), sensilla coeloconica (SC) and sensilla ampullacea (SA). The inner surface of the maxilla is filled with sensory folds and sensilla trichoidea (Figs. $2 \mathrm{M}-\mathrm{N}$ ).

\subsubsection{Sting apparatus}

Sting apparatus is well sclerotized with a straight sting shaft and tapers evenly to the bifurcated apical structure with basal musculature (Figs. 2 O).

\subsubsection{Sensilla of sting apparatus}

The dissection of the last abdominal segment of ant reveals the presence of sensilla trichoidea (ST-I, ST-II, ST-III), sensilla trichoidea curvata (STC) and sensilla basiconica (SB). STI are long, curved anteriorly, while sensilla ST-II and ST-III are smaller than ST-I and the ST-II are arranged throughout the external surface. ST-III are smallest sensilla present adjacent to the ST-II (Figs. 2 O).

\subsection{Host selection and Hunting Behavior}

The foraging habit, of $M$. brunnea (workers), involves movement in a sinuous path with widely opened antennae. During the host searching behavior of ant, there may be an involvement of the sensilla present on their antenna. The attack and feeding pattern of this ant is very aggressive: initially, one or very few ants attack the larva of $A$. mylitta and then other members of the colony joined the group for feeding (as described earlier [11]). As the feeding proceeds, the ants tear the host larva with their strong mandibles. The resulting oozing out of the haemolymph attracts other ants perceived by the chemoreceptors on the mouthparts. Then it commences the constant biting and pricking, in which the excess amount of hemolymphoozes out eventually causing larval mortality. During the predatory attack, the tasar host larvae try to escape, but the intensity of injuries and continuous biting by the 
ants make the larva defenseless. Moreover, we believe that the ant may spray their sting venom on the skin including injury site of the host larva which may cause irritation. During the predation, the predatory ant employs all the sensory inputs to accomplish the predatory success.

\section{DISCUSSION}

The predatory interaction between the predator Myrmicaria brunnea (Saunders) and the larvae of the tasar silkworm, Antheraea mylitta (Drury) was studied [6,7,11]. The workers of M. brunnea are aggressive over the first to the fifth larval stages of A. mylitta. Sometimes, the ants carry their prey to their ground nest either after cutting into small pieces or/and the whole prey including the fourth/fifth instar larvae of A. mylitta. The predatory activity of $M$. brunnea is found to be similar to the weaver ant, $O$. smaragdina $[17,18]$ on the tasar silkworm. The ant M. brunnea (Saunders) $[12,13]$ and its geniculate type of antennae is a characteristic of aculeate Hymenoptera [14-16]. A ball-like scape at the base region, present in the ants, Diacamma sp. and in Camponotus japonicus Mayr [14,15] are also observed in $M$. brunnea. The pedicel in M. brunnea is long and broad with an imbricate surface and covered with patches of sensilla, similar to C. japonicus and C. sericeus $[15,16]$ and in C. compressus [19]. In most of the ant species, the mouthparts are adapted for grasping and feeding the prey. While the receptors of taste are situated in the lower pair of jaws in the ants, which distinguish the different taste of fluids [20-22]. In ants, the mouthparts are equipped with mechano and chemoreceptors [22,23]. The mandibles in $M$. brunnea are powerful tools for catching prey, fighting, digging, seed crushing, wood-scraping, grooming, brood care and trophallaxis [23,24]. On the dorsal side of the mandibles, it possesses trichoid sensilla, which are densely distributed, whereas, on the ventral side, sensilla basiconica predominate. SB are also found on worker mandibles. The trichoid sensilla and small peglike sensilla basiconica, on the dorsal and ventral surface of mandibles observed are similar to earlier reports [25-27]. Various types of antennal sensilla have previously been reported in ants, Lasius fuliginosus (Latreille) [20] and Diacamma sp. [14, 28]. The present study reveals the diversity within each of the four basic types of antennal sensilla of M. brunnea, viz. the SB, ST, STC, and SC. Similarly, the presence of two subtypes of SB and three types of ST are reported in C. compressus [19] and other Hymenoptera $[14-16,20,29,30]$. Sensilla trichoidea are located on the antennae of $M$. brunnea at the pedicel region have also been reported in other species [14-16]. The SB on the antennae exhibit similar morphological structure to previously-studied ant species and may function as contact gustatory receptors $[15,28,31]$. The antennal basiconic sensilla (SB) of fire ants, Solenopsis invicta are also known to function as contact chemoreceptors [32,33]. Nakanishi et al. [15] categorized two types of trichoid sensilla along with the sensilla trichoidea curvata in $C$. japonicus which does not always respond to stimulation by alarm pheromones [31,34]. Thus, these may have a similar function in $M$. brunnea also. The STC in $M$. brunnea resembles those in other ant species $[15,35]$ which may perform as a contact chemosensory function $[15,36]$. The presence of ST, SB, and STC on the antenna of M. brunnea may perform similar function as reported earlier. In several Myrmicinae, moderately stipulated sting apparatus are well described [37-39] and are spatula-shaped in M. opaciventris. During predation, these ants deposit the venom into the prey's cuticle by wagging the bent gaster [40]. The furcula, a wishbone-shaped sclerite whose ventral arms are flexible, are attached to the base of the sting, causing the aculeus to pitch, roll, and yaw in probing for a sting site [41]. The behavior of insects is largely stereotype and is regulated by sensory inputs. Sensory capabilities, therefore, are key determinants of insect behavior. The olfactory sensilla are aggregated on the antennae and mouthparts bear gustatory sensilla. Commonly occurring olfactory sensilla are sensilla basiconica, sensilla trichoidea, sensilla coeloconica and sensilla ampullaceum and subtypes of these sensilla are also known [42]. Similarly, the diversity of antennal and mouthpart sensilla in $M$. brunnea regulates the feeding behavior of $M$. brunnea. The vigorous predatory host feeding activity in $M$. brunnea shows close resemblance with a well-known tasar larval predator, $O$. smaragdina [18]. Also, the occurrence of the predator depends on the variability in abiotic factors like temperature, relative humidity, and rainfall. The abundance of $M$. brunnea in tasar rearing fields causes a loss in tasar silk production. Therefore, to increase wild silk production, the population of this predatory ants needs to be extruded from tasar rearing fields.

\section{CONCLUSION}

The ant, M. brunnea attacks the larvae of the tropical tasar silkworm, A. mylitta. The predatory feeding behavior results in larval mortality of silkworm. In this study, we report the predatory feeding behavior, necessarily involves sensory inputs. Therefore, the control of this predator from the tasar rearing fields is needed. Hence, the present study may help to explore the further research intentions based on this neural mechanism and volatile cues which results mortality of the silkworms.

\section{ACKNOWLEDGEMENTS}

We thank the Directorate of Sericulture, Government of Maharashtra, India for allowing us to access tasar rearing fields in Vidarbha.We also thank the Director of Visvesvaraya National Institute of Technology (VNIT), Nagpur for SEM studies.

\section{REFERENCES}

1. Jolly MS, Chaturvedi SM, \& Prasad SA. Survey of Tasar crops in India. Indian Journal of Sericulture. 1968; 1: 50-58.

2. Jolly MS, Sen SK, Sonwalkar, TN, \& Prasad GK. Non-mulberry silks. Food and Agriculture Organization of United Nations, Service Bulletin. 1979;29, 1-178.

3. Jolly MS Package of practices for tropical tasar culture, Ranchi. Central Tasar Research Station, (Central Silk Board, Bombay). 1976; pp. 32.

4. Chatterjee KK, Chandra H, Gupta R, \& Chakravorty D. Tasar culture an approach of social upliftment of forest dwellers of Achanakmar 
Amarkantak Biosphere Reserve. Proceedings of workshop held at Tropical Forest Research Institute, Jabalpur on Research needs for Achanakmar- Amarkantak Biosphere Reserve 2007.

5. Singh RN, \& Thangavelu K. Parasites and predators of tasar silkworm Antheraea mylitta has many enemies. Indian Silk. 1991; pp. 33-36.

6. Gathalkar, GB and Barsagade, DD. Parasites-predators: their occurrence and invasive impact on the tropical tasar silkworm Antheraea mylitta (Drury) in the Zone of Central India. Current Science. 2016; 111 (10):1643-1657.

7. Barsagade DD. \& Gathalkar, GB. Myrmicaria brunnea (Saunders) (Hymenoptera: Formicidae): a new predator in tasar sericulture. Proceedings of the $10^{\text {th }}$ ANeT International Conference. Department of Zoology and Environmental Management, University of Kelaniya (Abstract). 2015; $\quad$ p. $53 . \quad$ URI: http://repository.kln.ac.lk/handle/123456789/10162

8. Bain A, Harrison RD, \& Schatz B. How to be an ant on figs. Acta Oecologica. 2014; 57:97-108.

9. Lach L, Parr C, \& Abbott K. Ant Ecology. Oxford University Press, Oxford. 2010.

10. General, DM, and Alpert, GD. A synoptic Review of the ant genera (Hymenoptera: Formicidae) of the Phillippines. Zookeys. 2012; 200: $1-111$.

11. Gathalkar, GB \& Barsagade: Predation potential of Myrmicaria brunnea (Hymenoptera: Formicidae) on the wild silkworm Antheraea mylitta (Lepidoptera: Saturniidae). (Communicated).

12. Bingham CT. Fauna of British India. Hymenoptera. 1903; Vol II. London.

13. Bakhtiar EY, Yamane SK, \& Maryati M. Morphological and behavioural characters of the two species-groups of the ant genus Myrmicaria (Insecta, Hymenoptera: Formicidae: Myrmicinae) from Southeast Asia. Species Diversity. 2009; 14, 249-265.

14. Okada Y, Miura, T, \& Tsuji K. Morphological differences between sexes in the ponerine ant, Diacamma sp. (Formicidae: Ponerinae). Sociobiology. 2006; 48: 527-541.

15. Nakanishi A, Nishino H, Watanabe H, Yokohari F, \& Nishikawa M. Sex-specific antennal sensory system in the ant Camponotus japonicus, structure and distribution of sensilla on the flagellum. Cell Tissue Research. 2009; 338: 79-97.

16. Mysore K, Subramanian, KA, Sarasij RC, Suresh A, Shyamala BV, Vijayraghavan K, and Rodrigues V. Caste and sex-specific olfactory glomerular organization and brain architecture in two sympatric ant species Camponotus sericeus and Camponotus compressus (Fabricius, 1798). Arthropod Structure and Development. 2009; 38: 485-497.

17. Offenberg J, Havanon S, Aksornkoae S, Macintosh DJ, \& Nielsen MG. Observations on the ecology of weaver ants Oecophylla smaragdina (Fabricius) in a Thai mangrove ecosystem and their effect on herbivory of Rhizophora mucronata Lam. Biotropica. 2004;36: 344-351.

18. Gathalkar GB. \& Barsagade DD. Predation biology of weaver ant Oecophylla smaragdina (Hymenoptera: Formicidae) in the field of tasar sericulture. Journal of Entomology and Zoology Studies. 2016; 4 (2): 07-10.

19. Barsagade DD, Tembhare DD, \& Kadu SG. Microscopic structure of antennal sensilla in the carpenter ant Camponotus compressus (Fabricius) (Formicidae: Hymenoptera), Asian Myrmecology. 2013; 5: 113-120.

20. Dumpert K. Alarm stoffrezeptoren auf der Antenne von Lasius fuliginosus (Hymenoptera: Formicidae). Zeitschrift für Vergleichende Physiologie. 1972;76: 403- 425.

21. Chapman RF. The insect structure and function $\left(4^{\text {th }}\right.$ ed.). Cambridge, Cambridge University Press. 1998.

22. Paul JP, Flavio R \& Hölldobler B. How do ants stick out their tongues? Journal of Morphology and Embryology. 2002; 254, 39-52.

23. Paul J. Mandible movements in ants. Comparative Biochemistry and Physiology. 2001; 13 (1): 7-20.
24. Hölldobler B, \& Wilson EO. The Ants. MA, Belknap Press of Harvard University, Cambridge, MA. 1990; pp. 732.

25. Zacharuk RY. Ultrastructure and Function of Insect Chemosensilla. Annual Review of Entomology, 1980; 25: 27-47.

26. Kapoor NN. Distribution and innervations of sensilla on the mouthparts of the Carnivorous stonefly nymph, Paragnetina media (walker) (Plecoptera: Perlidae). Canadian Journal of Zoology. 1989; 67 (4): 831- 38.

27. Barsagade DD, Tembhare DB, \& Kadu SG. SEM structure of mandibular sensilla in the carpenter ant Camponotus compressus (Fabricius) (Formicidae: Hymenoptera) Halters. 2010; 2 (1): 53-57.

28. Ozaki M, Wada-Katsumata A, Fujikawa K, Iwahasi M, Yokahari F, Satoji Y, Nishimurat, \& Yamaoka Y. Ant nestmate and nonnestmate discrimination by a chemosensory sensillum. Science. 2005;309: 311-314

29. Esslen J,\& Kaissling KE.: Zahl-und Verteilung antennaler Sensillen bei der Honigbiene (Apis mellifera L.). Zoomorphology. 1976; 83: 227-251.

30. Zacharuk RY. Antennae and sensilla. In Comprehensive Insect Physiology, BiochemistryandPharmacology. Vol. 6. Pergamon Press. Nervous System. Sensory Ed., Kerkut GA. \& Gilbert, LI. 1985; pp. 1- 69.

31. Mysore K, Shyamala BV, Rodrigues V. Morphological and developmental analysis of peripheral antennal chemosensory sensilla and central olfactory glomeruli in worker caste of Camponotus compressus (Fabricius, 1787). Arthropod Structure and Development. 2010; 39: 310-321.

32. Renthal RD. Sensory reception in fire ants, imported fire ant research and management project, Final report, Texas. 2003; pp. 1-3.

33. Renthal R, Velasqueza D, Olmosa D, Hamptona J, and Wergin WP. Structure and distribution of antennal sensilla of the red imported fire ant. Micron. 2003; 34: 405- 413.

34. Nakanishi A, Nishino H, Watanabe H, Yokohari F, and Nishikawa M. Sex-specific antennal sensory system in the ant Camponotus japonicus, glomerular organizations of antennal lobes. Journal of Comparative Neurology. 2010; 518: 2186-2201.

35. Hashimoto Y. Unique features of sensilla on the antennae of Formicidae (Hymenoptera). Applied Entomology and Zoology. 1990; 25: 491-501.

36. Altner $\mathrm{H}$, \& Prillinger L. Ultrastructure of invertebrate chemo-, thermo- and hygroreceptors and its functional significance.In, Bourne, G.H., Danielli, J.F. (Eds.), International Review of Cytology. Academic Press. 1980; pp. 69-139.

37. Kugler C. Evolution of the sting apparatus in the myrmicine ants. Evolution. 1979; 33: 117-130.

38. Kugler C. Stings of ants of the tribe Pheidologetini (Myrmicinae). Insecta Mundi. 1986; 1 (4): 221-230.

39. Kaib M, \& Dittebrand $H$. The poison gland of the ant Mvrmicariaeumenoides and its role in recruitment communication. Chemoecology. 1990; 1: 3-11.

40. Kenne M, Schatz B, Durand JL, \& Dejean A. Hunting strategy of a generalist ant species proposed as a biological control agent against termites. Entomologia Experimentalis et Applicata. 2000; 94: 31-40.

41. Kugler C. A comparative study of the myrmicine sting apparatus (Hymenoptera: Formicidae) Studia Entomologica. 1978; 20; 413 548.

42. Babu MJ, Ankolekar SM, \& Rajashekhar KP. Castes of the weaver ant Oecophylla smaragdina (Fabricius) differ in the organization of sensilla on their antennae and mouthparts. Current Science. 2011; 101 (6): 1-10.

\section{How to cite this article:}

Gathalkar GB, Barsagade DD. Cephalic Microstructure and its role in Predation Biology of Myrmicaria brunnea on Antheraea mylitta. J App Biol Biotech. 2018; 6(1): 1-6. 